\title{
Long-Term Left Ventricular Remodelling in Rat Model of Nonreperfused Myocardial Infarction: Sequential MR Imaging Using a 3T Clinical Scanner
}

\author{
Muhammad G. Saleh, ${ }^{1}$ Sarah-Kate Sharp, ${ }^{2}$ Alkathafi Alhamud, ${ }^{1}$ Bruce S. Spottiswoode, ${ }^{1,3}$ \\ Andre J. W. van der Kouwe, ${ }^{4,5}$ Neil H. Davies, ${ }^{2}$ Thomas Franz, ${ }^{2,6,7}$ and Ernesta M. Meintjes ${ }^{1}$ \\ ${ }^{1}$ MRC/UCT Medical Imaging Research Unit, Department of Human Biology, University of Cape Town, \\ Observatory 7925, South Africa \\ ${ }^{2}$ Cardiovascular Research Unit, Chris Barnard Division of Cardiothoracic Surgery, University of Cape Town, \\ Observatory 7925, South Africa \\ ${ }^{3}$ Division of Radiodiagnosis, Stellenbosch University, Matieland 7602, South Africa \\ ${ }^{4}$ Athinoula A. Martinos Center for Biomedical Imaging, Massachusetts General Hospital, Charlestown, MA 02129, USA \\ ${ }^{5}$ Department of Radiology, Harvard Medical School, Boston, MA 02115, USA \\ ${ }^{6}$ Centre for Research in Computational and Applied Mechanics, University of Cape Town, Rondebosch 7701, South Africa \\ ${ }^{7}$ Centre for High Performance Computing, Rosebank 7700, South Africa
}

Correspondence should be addressed to Muhammad G. Saleh, mgsaleh7@gmail.com

Received 6 March 2012; Accepted 11 June 2012

Academic Editor: M. Piacentini

Copyright (C) 2012 Muhammad G. Saleh et al. This is an open access article distributed under the Creative Commons Attribution License, which permits unrestricted use, distribution, and reproduction in any medium, provided the original work is properly cited.

Purpose. To evaluate whether 3T clinical MRI with a small-animal coil and gradient-echo (GE) sequence could be used to characterize long-term left ventricular remodelling (LVR) following nonreperfused myocardial infarction (MI) using semi-automatic segmentation software (SASS) in a rat model. Materials and Methods. 5 healthy rats were used to validate left ventricular mass (LVM) measured by MRI with postmortem values. 5 sham and 7 infarcted rats were scanned at 2 and 4 weeks after surgery to allow for functional and structural analysis of the heart. Measurements included ejection fraction (EF), end-diastolic volume (EDV), endsystolic volume (ESV), and LVM. Changes in different regions of the heart were quantified using wall thickness analyses. Results. LVM validation in healthy rats demonstrated high correlation between MR and postmortem values. Functional assessment at 4 weeks after MI revealed considerable reduction in EF, increases in ESV, EDV, and LVM, and contractile dysfunction in infarcted and noninfarcted regions. Conclusion. Clinical 3T MRI with a small animal coil and GE sequence generated images in a rat heart with adequate signal-to-noise ratio (SNR) for successful semiautomatic segmentation to accurately and rapidly evaluate long-term LVR after MI.

\section{Introduction}

Assessment of ventricular function and mass is an important guide for predicting survival in patients suffering from different cardiovascular diseases, such as ischemic heart disease [1]. In addition, the efficacy of long-term drug therapy of compounds such as angiotensin-converting enzyme (ACE) inhibitor and beta-adrenergic blocking agents are evaluated by assessment of left ventricular (LV) mass and function [2]. Rat models have been widely used to study LV performance and remodelling after induction of myocardial infarction (MI) $[3,4]$. In addition, the effects of ACE inhibitors and captopril have been examined in this way $[1,5]$. Hence, noninvasive methods to accurately quantify ventricular mass and function are invaluable to clinical and preclinical research.

Cardiac magnetic resonance imaging has been considered the optimal tool for noninvasive evaluation of LV function and mass due to its ability to acquire images with high spatial resolution and superb soft tissue contrast without risk of radiation [6-8]. Also, it allows for noninvasive imaging on 
the same animal over a prolonged period of time, providing subject-specific longitudinal data and reducing the number of animals needed.

Rodent (rat and mouse) models are preferred over large animal models because of their small size, easy availability and low maintenance cost. Furthermore, transgenic and gene-knockout technologies allow design and generation of rodents that mimic human cardiovascular disease [9]. However, rat models of heart failure are widely and successfully used and are preferred over mouse models because of reasons explained by Patten and Hall-Porter [10]: it is easier to perform open-chest surgical procedures and invasive hemodynamic assessments, and a greater number of postmortem histological or molecular biological analyses can be done given the approximately 10 -fold greater myocardial mass of rats compared with mice.

Imaging rats is challenging due to their small heart size and rapid heart rates [11]. Thus, most of these studies are conducted in high-field nonclinical magnetic resonance (MR) scanners with high performance gradient systems and small RF coils for rapid and high signal-to-noise ratio (SNR) imaging. Several articles have reported assessment of LV function in rats and mice using dedicated high-field MR scanners ( $\geq 4.7$ Tesla) [12-17]. However, these scanners are not available at many institutions [18]. Although cardiac imaging in rodents using clinical grade scanners has been implemented using optimized radio frequency (RF) coils, the images produced suffer from reduced signal and contrast due to lower field strength [17]. Consequently, high-resolution images $(<250 \mu \mathrm{m}$ in-plane resolution) acquired in clinical scanners with gradient echo (GE) sequence generally result in poor definition of the endocardial and epicardial boundaries, requiring manual intervention during the segmentation process $[19,20]$.

The purpose of this study was to assess whether $3 \mathrm{~T}$ clinical MRI with a small animal coil and GE sequence could generate images with sufficient contrast and resolution to characterize using semi-automatic segmentation software (SASS), long-term LV remodelling (LVR), and changes in functional parameters-such as LV ejection fraction (EF), end-diastolic (ED), and end-systolic (ES) volume-following induction of nonreperfused $\mathrm{MI}$ in rats.

\section{Materials and Methods}

2.1. Magnet and Gradient System. All scans were performed on a MAGNETOM Allegra 3T MRI scanner (Siemens, Erlangen, Germany). This system is equipped with maximum gradient strength of $40 \mathrm{mT} \cdot \mathrm{m}^{-1}$ and slew rate of $400 \mathrm{mT} \cdot \mathrm{m}^{-1} \cdot \mathrm{ms}^{-1}$. A custom built, circularly polarized, $70 \mathrm{~mm}$ (inner diameter) birdcage radiofrequency (RF) coil was used to transmit and receive the signal.

2.2. Animal Model. All animal studies were approved by the Faculty of Health Sciences Animal Research Ethics Committee (REC) and performed in accordance with the National Institutes of Health (NIH, Bethesda, MD, USA) guidelines. 17 male outbred Wistar albino rats were used for the study that weighed $195.3 \pm 10.8 \mathrm{~g}$ (standard deviation) at implant. The animals were split into three groups, namely, healthy/control $(n=5)$, sham $(n=5)$, and MI $(n=7)$. In the MI group, a nonreperfused MI was induced as previously described by Dobner et al. [21]. Briefly, rats were placed in an induction chamber and anesthetized with air flow containing 5\% isoflurane and $1.5 \%$ Oxygen. Animals were then intubated with a $16 \mathrm{G}$ catheter and mechanically ventilated with $1.5-2 \%$ isoflurane and $0.3 \%$ oxygen. A left intercostal thoracotomy was performed and MI was induced by permanent ligation of the left anterior descending (LAD) coronary artery. A 6-0 prolene suture was used to ligate and placed 2 to $3 \mathrm{~mm}$ below the left atrial appendix. Fibrillation and ischemia of the LV wall confirmed the infarction. Sham animals were subjected to the same procedure without ligation of LAD coronary artery. Following the surgical procedure, an analgesic dose of $100 \mu \mathrm{L}$ Temgesic was administered by intramuscular injection to the hind leg. Animals were monitored daily for the duration of the study. Analgesic (100 $\mu \mathrm{L}$ Temgesic) was administered every morning for $48 \mathrm{hrs}$ after surgery. All animals survived surgery.

\section{MR Image Acquisition}

3.1. Animal Preparation. Baseline imaging using healthy animals $(n=5)$ was performed with a GE fast low-angle shot $($ FLASH $)$ sequence $[4,17]$. Infarcted and sham rats were imaged 2 and 4 weeks after surgery. Before scanning, anaesthesia was induced and maintained through a nose cone containing an atmosphere of $1.5 \%$ isoflurane in air. Subdermal electrocardiogram (ECG) needle electrodes were attached on the right front and left rear legs. Respiration sig-nal was superimposed on the measured ECG signal by closely coupling one of the ECG lead wires to the rat's abdomen. A rectal temperature probe was used to measure the rat's core body temperature, which was maintained by circulating hot water through a tube in the rat bed. ECG, respiration, and core body temperature were monitored by a dedicated small animal MR-compatible monitoring and gating system (SA Instruments, Stony Brook, NY, USA).

3.2. MR Parameters. MR parameters for short-axis images were field of view $(\mathrm{FOV})=60 \mathrm{~mm}$, matrix size $=256 \times$ 256, in-plane resolution $234 \mu \mathrm{m}$, slice thickness $1.0 \mathrm{~mm}$, TR $7.7 \mathrm{~ms}$, TE $3.5 \mathrm{~ms}$, flip angle $25^{\circ}$ to $40^{\circ}$, and 3 signal averages. For the four-week scan, FOV and matrix size were increased to $75 \mathrm{~mm}$ and $320 \times 320$, respectively. In all cases, the FLASH sequence was triggered by both ECG and respiration. One k-space line was acquired per frame resulting in 18 to 30 frames per cardiac cycle depending on the heart rate. Three averages were implemented to yield adequate SNR. Acquisition time was approximately 2.5 minutes per slice, and 15 to 16 slices were typically required to cover the entire LV. One to two additional slices were acquired to cover the entire LV of the MI group at the 4-week scan due to animal growth. 


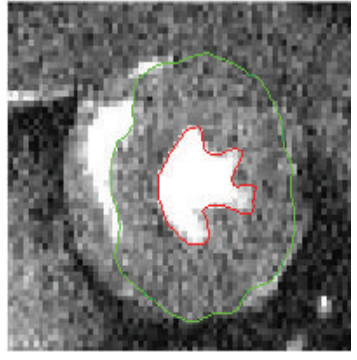

(a)

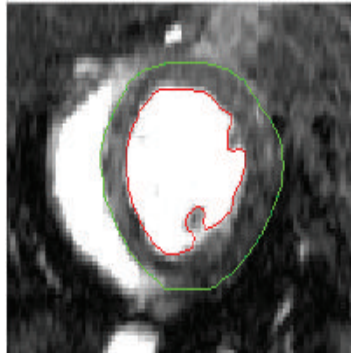

(b)

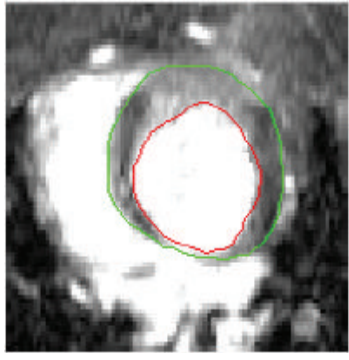

(c)

FIgure 1: Myocardial segmentation. Green line marks the epicardial border while red line marks endocardial border. ES (a) and ED (b) images illustrate papillary muscles segmented as part of myocardium. The last image (c) illustrates the straight line used to connect the two ends of the myocardium.

\section{Histological Preparation and Infarct Size Measurement}

A day after the completion of MRI scanning, all animals were euthanized with intraperitoneally injected sodium pentobarbitone whilst under isoflurane-induced anaesthesia. Hearts excised from healthy rats were trimmed free of right ventricle and atria to determine true LV mass. In the MI group, infarct size was determined histologically as described by Dobner et al. [21]. Briefly, the hearts were injected with $1 \mathrm{~mL}$ ice-cold saturated $\mathrm{KCl}$ solution after anaesthesia to arrest them in diastolic state and then excised for weighing. $3 \mu \mathrm{m}$ sections were obtained from 4 equal divisions of every heart. Each section was stained with Masson's trichrome to determine the extent of infarction and images captured with a Nikon E1000 M camera (Nikon, Tokyo, Japan) with a $0.5 x$ magnification lens. Infarct size was determined using the midline method as previously described by Takagawa et al. [22]. Briefly, infarct size was measured as the sum of midline length of infarcted myocardium from all sections divided by the sum of midline LV circumferences from all sections multiplied by 100. Measurements were acquired with Visiopharm Integrator Systems (Visiopharm, Hørsholm, Denmark).

\section{MR Image Analysis}

The image stacks with largest LV cavity were used as the end-diastolic (ED) images and the smallest as end-systolic (ES) images. From ED and ES short-axis images, LV borders were segmented using SASS, called Segment (http:// segment.heiberg.se/) without any long-axis correction. This software has been shown to yield low interobserver and intraobserver variability $(<5 \%)$ in volumetric analyses [23]. We implemented the LV segmentation technique and definitions for the most apical and basal slice that were previously explained by Riegler et al. [23]. Briefly, the most apical slice is defined as the first slice with no blood pool while the most basal slice is the one that shows a remaining part of the thick myocardium and is below the aortic valve. Papillary muscles were segmented as part of the myocardium (Figures 1(a) and $1(\mathrm{~b}))$.
The segmentation method employed in Segment software is based on the concept of deformable models, but extended with an enhanced and fast edge detection scheme [24]. Analyses using Segment software was initiated using the "left ventricular tool" algorithm. First, a marker was positioned in the center of the LV blood pool, from which the algorithm fits a deformable model that expands toward the endocardial border to include the LV blood pool across the various short-axis slices. Afterward, manual corrections were performed as necessary for the epicardial border, and for the endocardial border where the papillary muscles appeared separated from the myocardium. If the myocardium moved out of the plane due to long-axis contraction, a straight line was drawn to merge the two visible ends of the myocardium (Figure 1(c)), as described by Barbier et al. [25].

ED volume (EDV), ES volume (ESV), and LV mass (LVM) were measured by SASS. Other functional parameters were calculated as follows: stroke volume $(\mathrm{SV})=\mathrm{EDV}-\mathrm{ESV}$, $\mathrm{EF}=100 \times \mathrm{SV} / \mathrm{EDV}$, and cardiac output $(\mathrm{CO})=\mathrm{SV} \times$ Heart Rate.

LV wall thickness (WTh) was measured 4 weeks after surgery using the same software. WTh analyses were performed on midventricular slices as explained by Ross et al. [15] and Ojha et al. [26]. Briefly, following the delineation of epicardial and endocardial borders, LV wall was divided into 16 equal sectors using the right anterior ventricular insertion as a reference point. 4 contiguous measurements were averaged to form 4 equal sectors of $90^{\circ}$ each. These four sectors were called anterior, lateral, posterior, and septal. Papillary muscles were not included in this analysis. Subsequent to wall thickness measurements, \% systolic wall thickening (\%WTh) was calculated as $100 \times$ (systolic wall thickness - diastolic wall thickness)/diastolic wall thickness.

\section{Statistical Analysis}

If not indicated otherwise, all data are specified as mean \pm standard error of the mean (SEM). Correlation and BlandAltman analyses [27] were used to examine the relation and agreement between MR LVM and post-mortem measurements. An unpaired two-tailed $t$-test was used to compare LV function and mass between sham and MI groups. $P<0.05$ was considered significant. 
TABLE 1: LV global parameters.

\begin{tabular}{lcccc}
\hline & & Week 2 & & Week 4 \\
Group & Sham & MI & Sham & MI \\
\hline EDV $(\mu \mathrm{L})$ & $425.6 \pm 22.4$ & $487.5 \pm 56.1$ & $443.2 \pm 18.2$ & $624.4 \pm 62.6^{*, \dagger}$ \\
ESV $(\mu \mathrm{L})$ & $104.0 \pm 8.9$ & $189.0 \pm 21.5^{*}$ & $121.0 \pm 8.6$ & $298.6 \pm 40.9^{*, \dagger}$ \\
SV $(\mu \mathrm{L})$ & $322.0 \pm 15.7$ & $298.5 \pm 35.9$ & $322.2 \pm 12.2$ & $325.9 \pm 28.6$ \\
CO $(\mathrm{mL} /$ minute $)$ & $112.0 \pm 7.2$ & $105.2 \pm 11.6$ & $119.0 \pm 5.2$ & $123.2 \pm 12.0$ \\
EF $(\%)$ & $75.7 \pm 1.2$ & $61.1 \pm 1.6^{*}$ & $72.8 \pm 1.2$ & $52.8 \pm 2.7^{*, \dagger}$ \\
True heart weight $(\mathrm{g})$ & & & $1.2 \pm 0.1$ & $1.5 \pm 0.11^{\dagger}$ \\
LVM by MRI $(\mathrm{mg})$ & $600 \pm 20.9$ & $664.2 \pm 26.7^{*}$ & $615.2 \pm 10.2$ & $712.1 \pm 16.2^{*, \dagger}$ \\
\hline
\end{tabular}

${ }^{*} P<0.05$ versus week 2 sham, ${ }^{\dagger} P<0.05$ versus week 4 sham.

\section{Results}

All 17 animals survived the duration of the study. LVM mass validation showed very close correlation between the two methods. Rats in the MI group underwent considerable LVR, such as a significant drop in EF and increase in LVM, EDV, and ESV as highlighted below.

7.1. LV Mass Authentication. Healthy rats were used to validate MR LV mass with the true wet LV mass found by measurement on an electronic weight balance a day after baseline imaging. The mean MR LV mass was $512.4 \pm 31.8 \mathrm{mg}$, while the mean wet LV mass was $509.6 \pm 28 \mathrm{mg}$, correlation 0.87 . Bland-Altman analysis revealed a mean difference of $2 \mathrm{mg}$ between the two methods with $95 \%$ confidence interval of $72 \mathrm{mg}$ (Figure 2).

7.2. Structural and Global Functional Analysis. Short axis images were analysed with Segment. The midventricular images (Figure 3) at ES phase and histological images (Figure 4) acquired from sham and infarcted rats illustrate LV dilation and wall thinning in the anterior and lateral regions of the heart. Dilation of ESV is evident by 2 and 4 weeks after surgery demonstrating contractile dysfunction (Figure 3). From post-mortem measurements, infarct size was found to be $27.2 \pm 2.6 \%$. Table 1 describes cardiac parameters of sham and MI animals after surgery.

At 2 weeks after surgery, EDV did not increase significantly (Figure 5(a)), while there was significant increase in ESV in the MI group (Table 1, Figure 5(b)) compared to the sham group $(P<0.05)$. There was no significant change in $\mathrm{CO}$ and SV at 2 and 4 weeks after surgery. At 4 weeks after surgery, there was significant enlargement of EDV and ESV in the MI rats compared to sham rats at 4 weeks aftert surgery $(P<0.05$, Table 1$)$. For the MI group, EF dropped significantly at 2 weeks and was even lower at 4 weeks after surgery (Table 1, Figure 5(c)). LV mass was significantly greater in MI rats compared to sham rats both at 2 and 4 weeks after surgery (Table 1, Figure 5(d)).

7.3. Regional Functional Analysis. The MI group revealed dyskinesia and severe wall thinning in the infarcted area (Figure 3) at 4 weeks after surgery. In order to quantify this, we performed WTh analyses for a midventricular slice

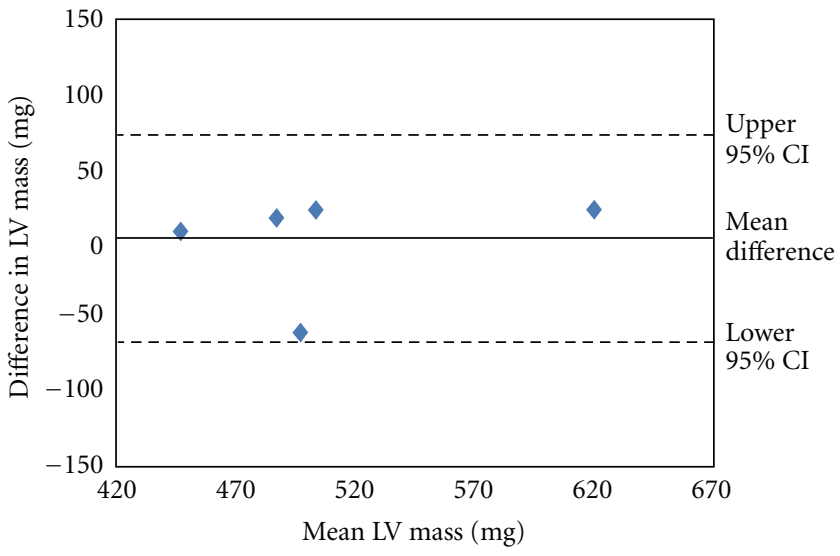

FiguRE 2: Bland-Altman analysis showing agreement between MR measurement and post-mortem measurements of LV mass in healthy rats. The analysis revealed mean difference of $2 \mathrm{mg}$ (solid line) and 95\% confidence interval of $72 \mathrm{mg}$ (mean difference $\pm 1.96 \times$ standard deviation). CI: confidence interval.

4 weeks after surgery in the MI and sham rats. At ED in the MI group, there was a significant drop in wall thickness in the anterior region (from $1.7 \pm 0.1 \mathrm{~mm}$ to $1.3 \pm 0.1 \mathrm{~mm}, P<0.05$ ), and a modest decrease in the lateral region (Figure $6(\mathrm{a})$ ). In addition, in the posterior region there was significant increase in wall thickness (from $1.6 \pm 0.04 \mathrm{~mm}$ to $2.0 \pm$ $0.1 \mathrm{~mm}, P<0.05)$ while there was modest increase in the septal region. Increases in the posterior and septal regions indicate the presence of hypertrophy. At ES in the MI group, there was a significant drop in wall thickness in the anterior (from $2.8 \pm 0.2 \mathrm{~mm}$ to $1.9 \pm 0.2 \mathrm{~mm}, P<0.05$ ) and lateral regions (from $3.3 \pm 0.1 \mathrm{~mm}$ to $2.2 \pm 0.3 \mathrm{~mm}, P<0.05$, Figure 6(b)), while there was a modest decrease in wall thickness in lateral and septal regions. Histological analysis of the MI group revealed complete absence of scar tissue in the septal region (Figure 4(a)). In contrast, most of the anterior and lateral quadrants of the heart demonstrate the presence of scar tissue and severe wall thinning (Figure 4(a)). These observations were in agreement with WTh analysis as these two quadrants suffered severe contractile dysfunction as a result of $\mathrm{MI}$, as evident from the significantly reduced wall thickening during systole (Figure 6(b)). 

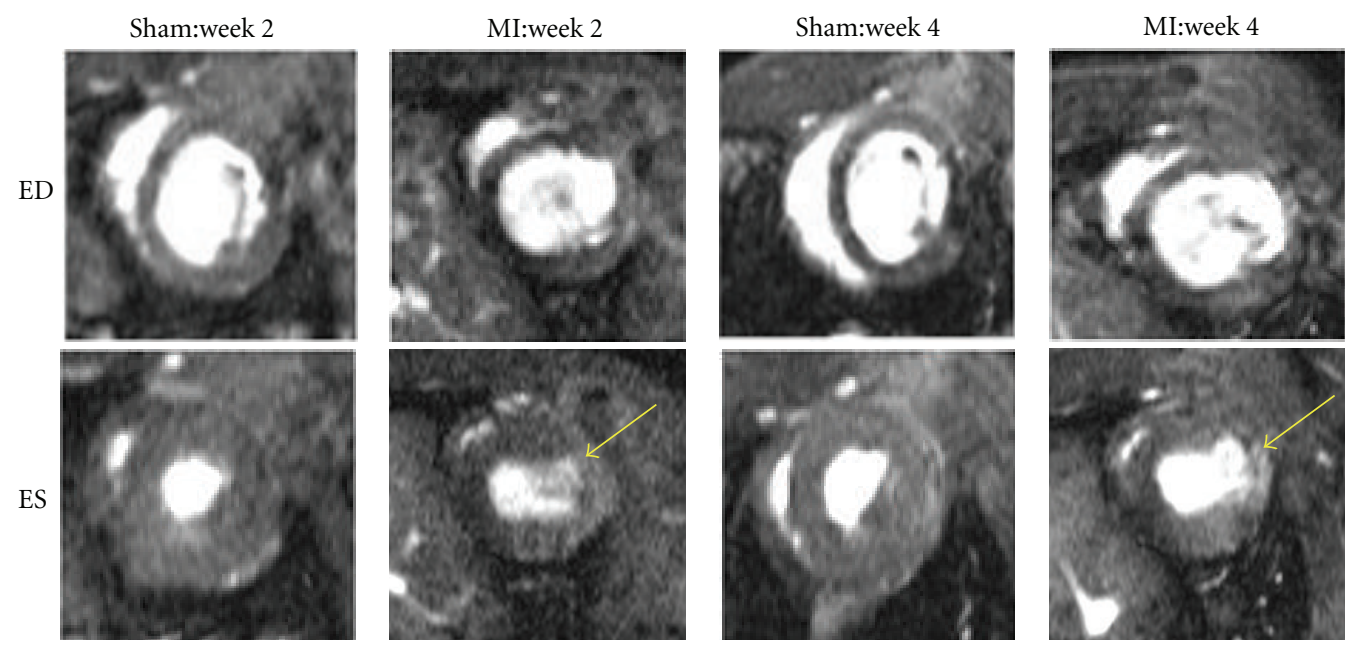

FIGURE 3: Short-axis images of the heart for sham and MI rats. Top row shows ED images 2 and 4 weeks after surgery, and bottom row shows the same for ES. ES images demonstrate significant dilation and wall thinning (arrows) in MI group at 2 and 4 weeks after surgery.

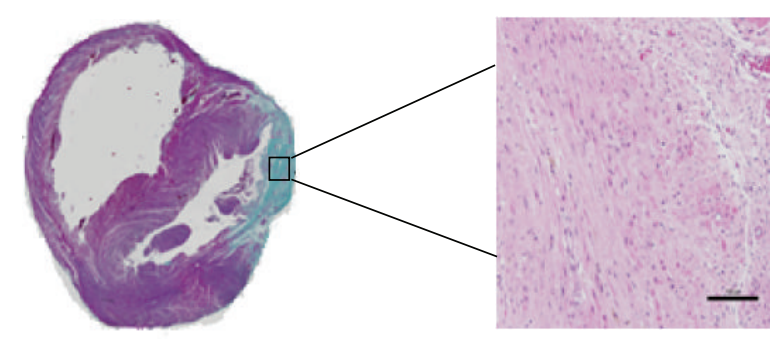

(a)

(b)

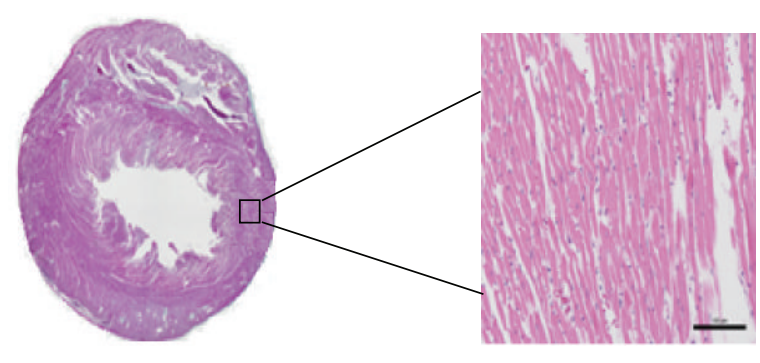

(c)

(d)

Figure 4: Comparison of histological images 4 weeks after surgery for an MI rat ((a), (b)) and a sham rat ((c), (d)). (a) Myocardial tissue slice of MI rat-blue stain corresponds to the infarcted area in the anterior and lateral regions of the heart. Significant wall thinning is evident in infarcted areas. (b) Hematoxylin-eosin-stained slice from an infarcted rat $\times 10$ magnification (scale bar $=100 \mu \mathrm{m}$ ). Loss of myocytes is evident in the infarcted area which is rich with atypical nuclei. (c) Myocardial tissue slice of a sham rat. Absence of blue stain confirms that LAD ligation caused myocardial infarction in infarcted rats. (d) Hematoxylin-eosin-stained slice from a sham rat $\times 10$ magnification (scale bar $=100 \mu \mathrm{m}$ ). Appropriate alignment and no loss of myocytes indicate absence of infarction.

\section{Discussion}

This study demonstrates serial assessment of rat heart, structurally and functionally, after nonreperfused MI using a 3T clinical MR scanner. The characteristics of LVR, such as LV dilation, hypertrophy, and wall thinning, were evident on these images. The implementation of the MR sequence parameters and a small animal coil yielded images with sufficient SNR and contrast to delineate major anatomical landmarks, such as papillary muscles. In addition, these parameters produced images with excellent spatial resolution within reasonable scan times. This level of the resolution made it possible to perform accurate quantitative WTh analyses, thereby providing extra information and better understanding of LVR in the rat heart.

Due to very low temporal resolution $(39 \mathrm{~ms})$, Franco et al. [20] performed multiple acquisitions to determine trigger delay to obtain ED and ES frames for functional analysis. 


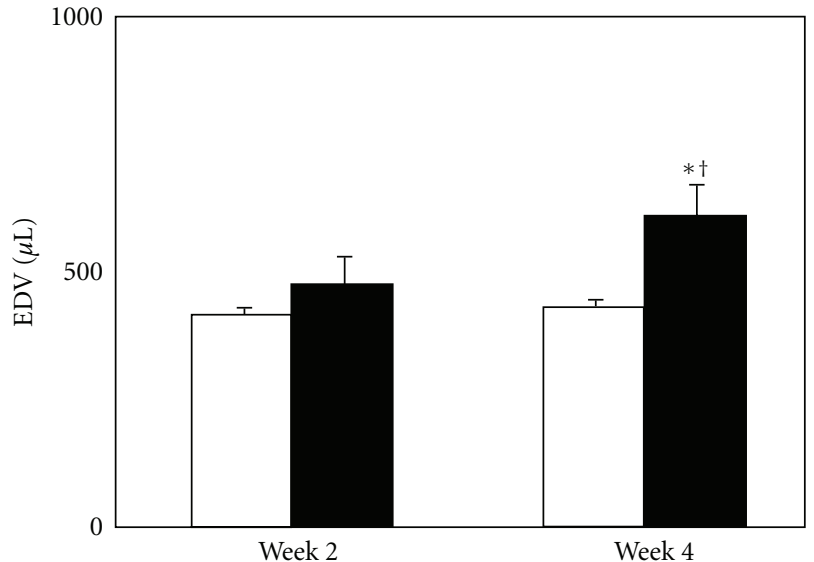

$\square$ Sham

(a)

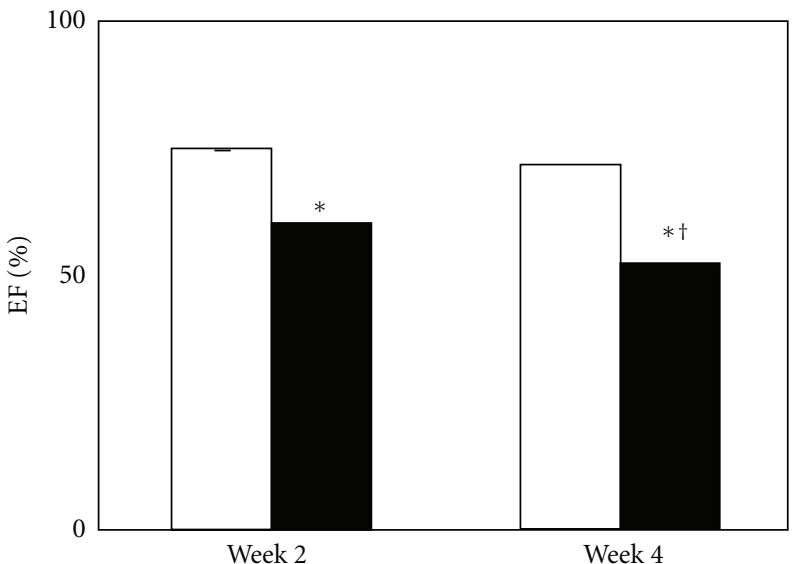

$\square$ Sham
MI

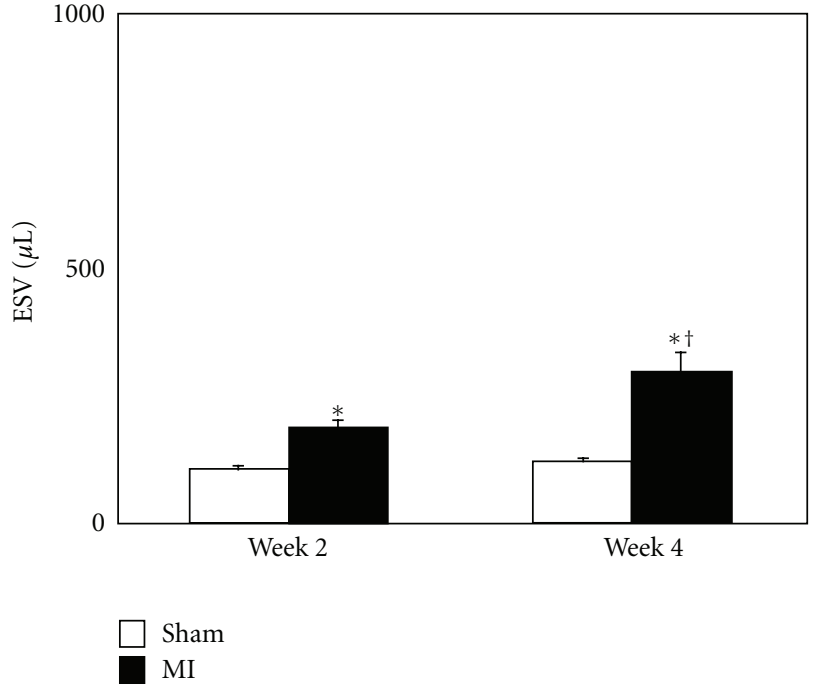

(b)

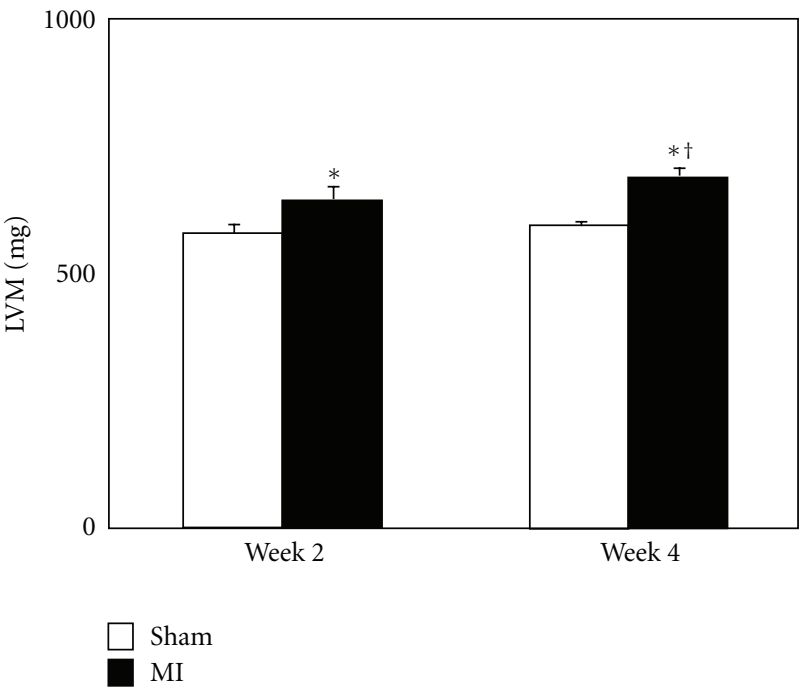

(d)

FIGURE 5: Average LV functional parameters and LVM determined by MRI. In the MI group, EDV (a) and ESV (b) were significantly higher at 4 weeks. ESV was also higher at 2 weeks compared to sham rats. EF (c) was significantly lower at both 2 and 4 weeks. LVM (d) was significantly higher at 2 weeks and 4 weeks in MI rats indicating hypertrophy. ${ }^{*} P<0.05$ versus week 2 sham, ${ }^{\dagger} P<0.05$ versus week 4 sham.

However, our temporal resolution $(7.7 \mathrm{~ms})$ was higher than previously reported values with clinical and non-clinical scanners [26, 28-30]. This resolution resulted in large number of frames per cardiac cycle which enabled us to obtain accurately ED and ES frames without the need of multiple acquisitions, resulting in reduction in scan time, and accurate global and regional functional analyses [20]. Furthermore, such high temporal resolution is necessary for depiction of LV dynamics in rats, especially with the heart rates of 300 beats per minute or above [31].

In our study, the results of LVM measured by MRI correlated well with post-mortem values. In addition, BlandAltman analysis revealed very low mean difference and low variation $(<15 \%$ of mean of true wet LVM) between the two methods. Moreover, these results are in close agreement with previous studies [16, 31]. MRI has been considered the gold standard for accurate measurements of LVM in small animal models in non-clinical scanners [17, 30, 32]. However, this study shows that accurate and reliable volumetric and mass measurements can also be performed on data acquired with a $3 \mathrm{~T}$ clinical scanner.

The contrast between LV blood pool and myocardium was large enough to effectively use SASS for segmentation to evaluate cardiac parameters. We compared our EF values, a major determinant of cardiac function, with reported values in the manner explained by Riegler et al. [23] and Delattre at al. [28]. In our study, EF in healthy animals $(71.8 \pm 1.3 \%)$ was equivalent to reported values $[4,33]$. Similarly, EF in sham rats showed slight variation but was equivalent to values reported by Nahrendorf et al. [34]. EF in the MI group at 


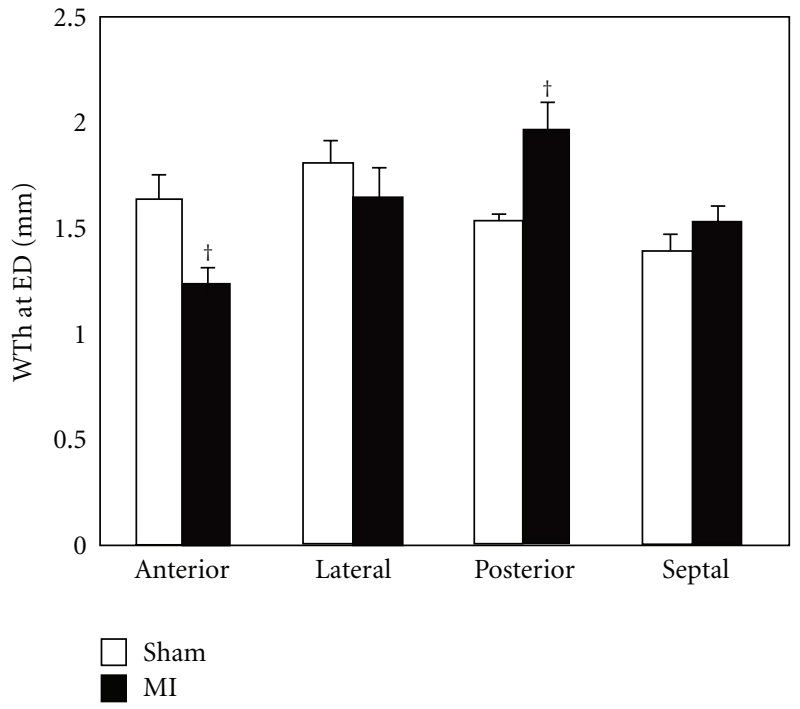

(a)

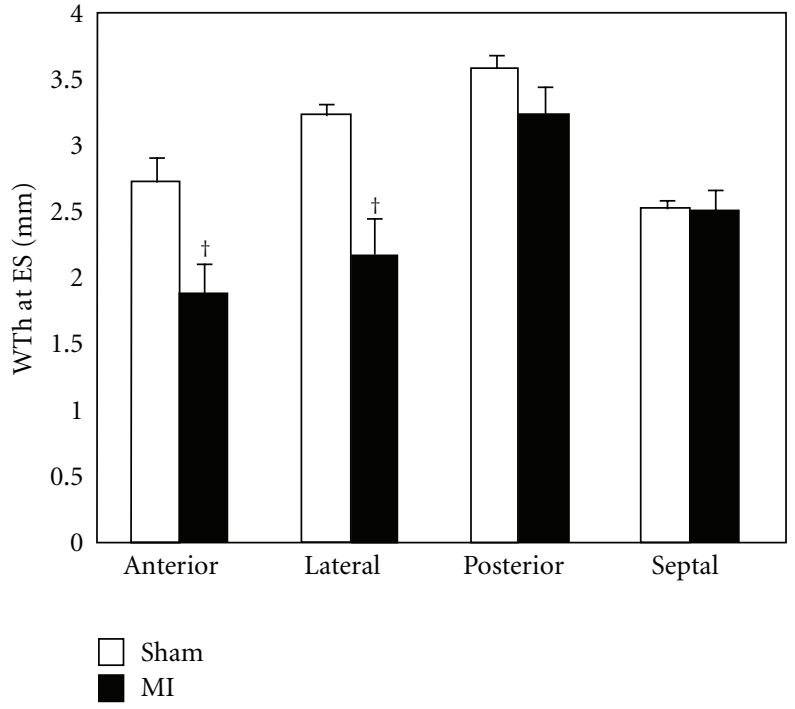

(b)

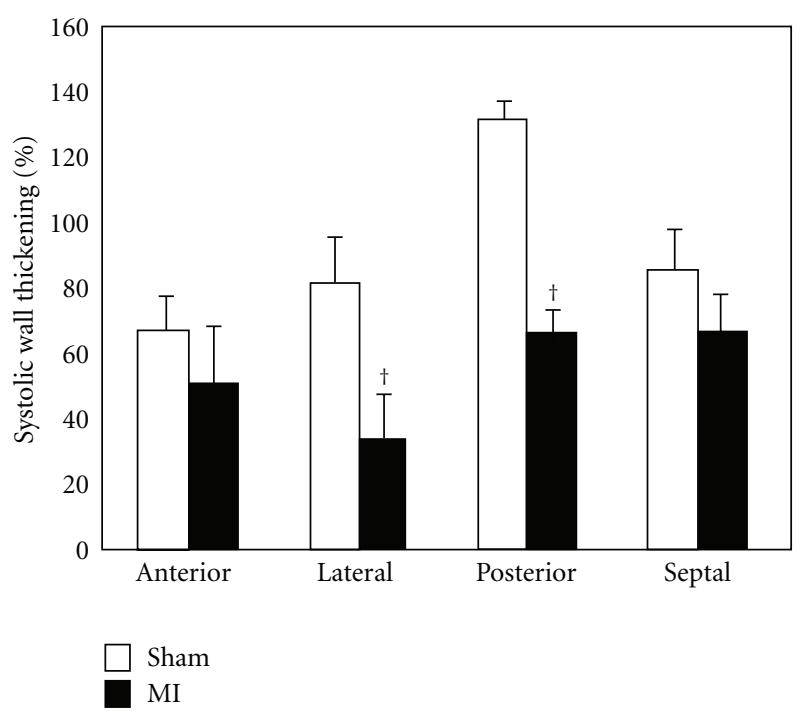

(c)

Figure 6: Analyses of regional LV function 4 weeks after surgery. Significant wall thinning occurs at ED in anterolateral regions while the posterior region demonstrates significant wall thickening (a). At ES, significant wall thinning is observed in anterior and lateral regions, while a modest decrease is evident in the posterior region (b). While all four regions show reduction in systolic wall thickening, it is only significant in the lateral and posterior regions (c). ${ }^{\dagger} P<0.05$ versus week 4 sham.

4 weeks was similar to the value reported by Carr et al. [35], but was higher than those reported by Stuckey et al. [36] and Nahrendorf et al. [4]. The variation in EF in infarcted rats most likely results from variation in infarct size, leading to differences in level of contractility [1].

During segmentation, some previous studies considered papillary muscles as part of the LV blood pool (not part of myocardium) $[7,37,38]$. This approach has been adopted due to its simplicity, as the shape of the LV blood pool is made much simpler, thus making the evaluation of LV volume faster and more time efficient [39]. However, omission of papillary muscles causes significant differences in measurements of EDV, ESV, and LVM, but not in EF
$[39,40]$. Nonetheless, possible effects of omitting papillary muscles should be taken into account when performing data analysis, especially during estimation of ESV [23]. In our study, papillary muscles were treated as part of myocardium because they contribute considerably to the blood pool at end diastole [40]. In addition, strong correlation between postmortem and MRI LVM can be achieved with the inclusion of these muscles in the LV myocardium [31, 40, 41].

The analysis time in our study ( $25 \pm 5$ minutes) was longer than previously reported values (10 and 8 minutes) using other SASS $[42,43]$. Nevertheless, these studies conducted cardiac analysis in mouse heart, thus the number of slices covering the whole heart with the same slice thickness 
was lower. Time savings for SASS are enormous, compared to manual segmentation. It can range between $20 \%$ and $70 \%$ depending on the number of cardiac frames segmented manually [23]. In our study, the segmentation time was significantly shorter than reported values using manual segmentation software (53 minutes and 2.5 hours) [23, 42], which led to fast and accurate volumetric analysis. The time savings allowed us to perform longitudinal studies and analyze cardiac parameters over time, such as EDV, ESV, EF, $\mathrm{SV}$, and CO. Also, it granted us the opportunity to perform regional functional analysis. Similar to a previous study [23], a significant amount of time was spent manually adjusting the contour line defining the epicardial border. Therefore, contrast enhancement between myocardium and surrounding tissues may lead to greater time savings.

In the sham group there was no significant variation in SV, CO, and LVM at either 2 or 4 weeks (Table 1). In the MI group, rats underwent LVR, in terms of functional and structural changes (Table 1), after LAD occlusion. The major functional change was a significant drop in EF due to the large extent of the fibrous tissue (loss of myocardium) causing contractile dysfunction in the heart (Figures 4(a) and $6(c))[3,44]$. The contractile dysfunction also caused significant increase in ESV (Figure 5(b)) at 2 weeks. Furthermore, there was a slight increase in EDV at 2 weeks, which would result in maintenance of SV [1]. The increase in ventricular volumes would likely amplify diastolic and systolic wall stress which can serve as a stimulus for further ventricular dilation [1]. Thus, further significant increase in ESV and EDV was observed 4 weeks after surgery. LV hypertrophy was evident in infarcted rats as indicated by severe increase in heart weight and LVM at both 2 and 4 weeks, probably due to progressive LV dilation (Table 1 and Figure 5(d)) [4]. In parallel with increase in LVM, LV hypertrophy was also evident in posterior and septal regions (Figure 6(a)) through WTh analysis. This was also observed by Litwin et al. [45] and Rubin et al. [46] due to increase in myocyte cell volume in attempts to stabilise contractile function and reduce progressive dilation [47].

In the MI group, collagen scarring was evident in anterior and lateral regions (blue-stained region) of the heart 4 weeks after surgery (Figure 4(a)). By contrast, rats in the sham group had no scarring (Figures $4(\mathrm{c})$ and $4(\mathrm{~d})$ ). This observation confirms that infarction was induced only by ligation of the LAD coronary artery in the MI group. In regions with infarction, fibroblasts are transformed into myofibroblasts which are responsible for collagen formation to stabilise distending forces and prevent further deformation [47]. By 3 to 4 weeks, the infarcted region is completely healed by formation of fibrous tissue (collagen scar) (Figure 4(b)) $[46,48]$. Regional functional analysis revealed significant reduction in wall thickness at ED in the anterior region and a modest decrease in the lateral region (Figure 6(a)). This was a result of necrosis of myocytes in infarcted regions [1]. At ES, WTh analysis revealed significant reduction in anterior and lateral regions and modest reduction in the remaining regions of the heart (Figure 6(b)). Furthermore, \%WTh revealed reduced wall thickening in all regions, which is significant only in lateral and posterior regions (Figure 6(c)).
These reductions in ES wall thickness and wall thickening arise due to contractile dysfunction, not only, in infarcted regions but also in non-infarcted regions $[16,49]$. However, anterior and lateral regions showed a greater deficit in function (Figures 6(a) and 6(b)) than other regions because of considerable loss of viable myocardium (Figure 4(b)). This loss also strongly affects functional parameters as explained above.

In summary, we have demonstrated assessment of a rat model of nonreperfused MI using a 3T clinical MRI and SASS, called Segment. Following the induction of MI, considerable changes in functional and structural parameters were found, such as a decrease in EF and increases in EDV, ESV, and LVM which were quantified effectively with SASS in a short period of time. These results are in good agreement with previous studies conducted with non-clinical scanners $[4,33,34]$. Furthermore, the high in-plane resolution of our images $(234 \mu \mathrm{m})$ enabled us to perform WTh analysis revealing important relationships between LV function and LVR.

In this study, ECG and respiratory triggering with 3 signal averages were necessary to acquire high-quality images. Some images, occasionally, suffered when rats had arrhythmic heartbeats. Due to long TE, some flow artefacts were observed, however those artefacts were far away from ED and ES frames, allowing robust and reliable functional and structural assessment of the rat heart. Hardware limitations and dephasing gradient lobes in the FLASH sequence limit the achievable reduction in TE, but implementation of spiral imaging can eradicate flow artefacts [50]. A large flip angle was used to provide high contrast between endocardium and the LV blood pool. Unfortunately, the epicardium did not benefit from this contrast phenomenon causing ambiguity in delineating the epicardial border. Riegler et al. [23] and Ross et al. [15] reported similar limitations due to the very small contrast difference between epicardium and surrounding tissue, and susceptibility differences between lung and the heart due to magnetic field inhomogeneities.

\section{Conclusion}

Clinical 3T MRI with a small animal coil and GE sequence generated images of rat heart with adequate SNR for successful semi-automatic segmentation to accurately and rapidly evaluate long-term LVR following nonreperfused MI.

\section{Acknowledgments}

The authors would like to thank Fulufhelo Masithulela for assistance with handling of the animals, Dr. Vijay Dahya for advice and guidance on image segmentation, Charles Harris for construction of the rat bed, Mogammad Fakier for construction of the automatic temperature regulation system, and Dr. Lawrence Wald and Azma Mareyam from the Martinos Center for Biomedical Imaging at Massachusetts General Hospital for guidance in building the small animal coil. This study was supported by the South African Research Chairs Initiative of the Department of Science and Technology and National Research Foundation of South Africa, 
the Medical Research Council of South Africa, and NIH Grant no. R21AA017410.

\section{References}

[1] M. A. Pfeffer and E. Braunwald, "Ventricular remodeling after myocardial infarction: experimental observations and clinical implications," Circulation, vol. 81, no. 4, pp. 1161-1172, 1990.

[2] P. Gaudron, C. Eilles, I. Kugler, and G. Ertl, "Progressive left ventricular dysfunction and remodeling after myocardial infarction: potential mechanisms and early predictors," Circulation, vol. 87, no. 3, pp. 755-763, 1993.

[3] M. A. Pfeffer, J. M. Pfeffer, and M. C. Fishbein, "Myocardial infarct size and ventricular function in rats," Circulation Research, vol. 44, no. 4, pp. 503-512, 1979.

[4] M. Nahrendorf, F. Wiesmann, K. H. Hiller et al., "Serial cinemagnetic resonance imaging of left ventricular remodeling after myocardial infarction in rats," Journal of Magnetic Resonance Imaging, vol. 14, no. 5, pp. 547-555, 2001.

[5] M. A. Pfeffer, J. M. Pfeffer, C. Steinberg, and P. Finn, "Survival after an experimental myocardial infarction: beneficial effects of long-term therapy with captopril," Circulation, vol. 72, no. 2, pp. 406-412, 1985.

[6] C. Badea, E. Bucholz, L. Hedlund, H. Rockman, and G. Johnson, "Imaging methods for morphological and functional phenotyping of the rodent heart," Toxicologic Pathology, vol. 34, no. 1, pp. 111-117, 2006.

[7] J. Barkhausen, S. G. Ruehm, M. Goyen, T. Buck, G. Laub, and J. F. Debatin, "MR evaluation of ventricular function: true fast imaging with steady-state precession versus fast low-angle shot cine MR imaging: feasibility study," Radiology, vol. 219, no. 1, pp. 264-269, 2001.

[8] L. A. Colby and B. J. Morenko, "Clinical considerations in rodent bioimaging," Comparative Medicine, vol. 54, no. 6, pp. 623-630, 2004.

[9] P. Balakumar, A. P. Singh, and M. Singh, "Rodent models of heart failure," Journal of Pharmacological and Toxicological Methods, vol. 56, no. 1, pp. 1-10, 2007.

[10] R. D. Patten and M. R. Hall-Porter, "Small animal models of heart failure development of novel therapies, past and present," Circulation, vol. 2, no. 2, pp. 138-144, 2009.

[11] B. Driehuys, J. Nouls, A. Badea et al., "Small animal imaging with magnetic resonance microscopy," ILAR Journal, vol. 49, no. 1, pp. 35-53, 2008.

[12] J. E. Schneider, T. Lanz, H. Barnes et al., "Ultra-fast and accurate assessment of cardiac function in rats using accelerated MRI at 9.4 Tesla," Magnetic Resonance in Medicine, vol. 59, no. 3, pp. 636-641, 2008.

[13] C. Chapon, J. S. Jackson, E. O. Aboagye, A. H. Herlihy, W. A. Jones, and K. K. Bhakoo, "An in vivo multimodal imaging study using MRI and pet of stem cell transplantation after myocardial infarction in rats," Molecular Imaging and Biology, vol. 11, no. 1, pp. 31-38, 2009.

[14] E. B. Schelbert, L. Y. Hsu, S. A. Anderson et al., "Late gadolinium-enhancement cardiac magnetic resonance identifies postinfarction myocardial fibrosis and the border zone at the near cellular level in ex vivo rat heart," Circulation, vol. 3 , no. 6, pp. 743-752, 2010.

[15] A. J. Ross, Z. Yang, S. S. Berr et al., "Serial MRI evaluation of cardiac structure and function in mice after reperfused myocardial infarction," Magnetic Resonance in Medicine, vol. 47, no. 6, pp. 1158-1168, 2002.
[16] Z. Yang, S. S. Berr, W. D. Gilson, M. C. Toufektsian, and B. A. French, "Simultaneous evaluation of infarct size and cardiac function in intact mice by contrast-enhanced cardiac magnetic resonance imaging reveals contractile dysfunction in noninfarcted regions early after myocardial infarction," Circulation, vol. 109, no. 9, pp. 1161-1167, 2004.

[17] A. N. Price, K. K. Cheung, J. O. Cleary, A. E. Campbell, J. Riegler, and M. F. Lythgoe, "Cardiovascular magnetic resonance imaging in experimental models," The Open Cardiovascular Medicine Journal, vol. 4, pp. 278-292, 2010.

[18] W. D. Gilson and D. L. Kraitchman, "Cardiac magnetic resonance imaging in small rodents using clinical 1.5-T and 3.0-T scanners," Methods, vol. 43, no. 1, pp. 35-45, 2007.

[19] F. Franco, G. D. Thomas, B. Giroir et al., "Magnetic resonance imaging and invasive evaluation of development of heart failure in transgenic mice with myocardial expression of tumor necrosis factor- $\alpha$, Circulation, vol. 99, no. 3, pp. 448-454, 1999.

[20] F. Franco, S. K. Dubois, R. M. Peshock, and R. V. Shohet, "Magnetic resonance imaging accurately estimates LV mass in a transgenic mouse model of cardiac hypertrophy," American Journal of Physiology, vol. 274, no. 2, pp. H679-H683, 1998.

[21] S. Dobner, D. Bezuidenhout, P. Govender, P. Zilla, and N. Davies, "A synthetic non-degradable polyethylene glycol hydrogel retards adverse post-infarct left ventricular remodeling," Journal of Cardiac Failure, vol. 15, no. 7, pp. 629-636, 2009.

[22] J. Takagawa, Y. Zhang, M. L. Wong et al., "Myocardial infarct size measurement in the mouse chronic infarction model: comparison of area- and length-based approaches," Journal of Applied Physiology, vol. 102, no. 6, pp. 2104-2111, 2007.

[23] J. Riegler, K. K. Cheung, Y. F. Man, J. O. Cleary, A. N. Price, and M. F. Lythgoe, "Comparison of segmentation methods for MRI measurement of cardiac function in rats," Journal of Magnetic Resonance Imaging, vol. 32, no. 4, pp. 869-877, 2010.

[24] E. Heiberg, L. Wigstrom, M. Carlsson, A. Bolger, and M. Karlsson, "Time resolved three-dimensional automated segmentation of the left ventricle," IEEE, pp. 599-602, 2005.

[25] C. E. Barbier, L. Johansson, L. Lind, H. Ahlström, and T. Bjerner, "The exactness of left ventricular segmentation in cine magnetic resonance imaging and its impact on systolic function values," Acta Radiologica, vol. 48, no. 3, pp. 285-291, 2007.

[26] N. Ojha, S. Roy, J. Radtke et al., "Characterization of the structural and functional changes in the myocardium following focal ischemia-reperfusion injury," American Journal of Physiology, vol. 294, no. 6, pp. H2435-H2443, 2008.

[27] J. M. Bland and D. G. Altman, "Statistical methods for assessing agreement between two methods of clinical measurement," The Lancet, vol. 1, no. 8476, pp. 307-310, 1986.

[28] B. M. A. Delattre, V. Braunersreuther, J. N. Hyacinthe, L. A. Crowe, F. Mach, and J. P. Vallée, "Myocardial infarction quantification with Manganese-Enhanced MRI (MEMRI) in mice using a 3-T clinical scanner," NMR in Biomedicine, vol. 23, no. 5, pp. 503-513, 2010.

[29] J. R. Jones, J. F. Mata, Z. Yang, B. A. French, and J. N. Oshinski, "Left ventricular remodeling subsequent to reperfused myocardial infarction: evaluation of a rat model using cardiac magnetic resonance imaging," Journal of Cardiovascular Magnetic Resonance, vol. 4, no. 3, pp. 317-326, 2002.

[30] A. Protti, A. Sirker, A. M. Shah, and R. Botnar, "Late gadolinium enhancement of acute myocardial infarction in mice at 7-T: cine-FLASH versus inversion recovery," Journal of Magnetic Resonance Imaging, vol. 32, no. 4, pp. 878-886, 2010. 
[31] J. Ruff, F. Wiesmann, K. H. Hiller et al., "Magnetic resonance microimaging for noninvasive quantification of myocardial function and mass in the mouse," Magnetic Resonance in Medicine, vol. 40, no. 1, pp. 43-48, 1998.

[32] S. E. Slawson, B. B. Roman, D. S. Williams, and A. P. Koretsky, "Cardiac MRI of the normal and hypertrophied mouse heart," Magnetic Resonance in Medicine, vol. 39, no. 6, pp. 980-987, 1998.

[33] D. Thomas, V. A. Ferrari, M. Janik et al., "Quantitative assessment of regional myocardial function in a rat model of myocardial infarction using tagged MRI," Magnetic Resonance Materials in Physics, Biology and Medicine, vol. 17, no. 3-6, pp. 179-187, 2004.

[34] M. Nahrendorf, K. H. Hiller, A. Greiser et al., "Chronic coronary artery stenosis induces impaired function of remote myocardium: MRI and spectroscopy study in rat," American Journal of Physiology - Heart and Circulatory Physiology, vol. 285, no. 6, pp. H2712-H2721, 2003.

[35] C. A. Carr, D. J. Stuckey, L. Tatton et al., "Bone marrowderived stromal cells home to and remain in the infarcted rat heart but fail to improve function: an in vivo cine-MRI study," American Journal of Physiology - Heart and Circulatory Physiology, vol. 295, no. 2, pp. H533-H542, 2008.

[36] D. J. Stuckey, C. A. Carr, D. J. Tyler, and K. Clarke, "Cine-MRI versus two-dimensional echocardiography to measure in vivo left ventricular function in rat heart," NMR in Biomedicine, vol. 21, no. 7, pp. 765-772, 2008

[37] R. J. M. Van Geuns, T. Baks, E. H. B. M. Gronenschild et al., "Automatic quantitative left ventricular analysis of cine MR images by using three-dimensional information for contour detection," Radiology, vol. 240, no. 1, pp. 215-221, 2006.

[38] A. A. Young, B. R. Cowan, S. F. Thrupp, W. J. Hedley, and L. J. Dell'Italia, "Left ventricular mass and volume: fast calculation with guide-point modeling on MR images," Radiology, vol. 216, no. 2, pp. 597-602, 2000.

[39] B. Sievers, S. Kirchberg, A. Bakan, U. Franken, and H. J. Trappe, "Impact of papillary muscles in ventricular volume and ejection fraction assessment by cardiovascular magnetic resonance," Journal of Cardiovascular Magnetic Resonance, vol. 6, no. 1, pp. 9-16, 2004.

[40] J. Vogel-Claussen, J. P. Finn, A. S. Gomes et al., "Left ventricular papillary muscle mass: relationship to left ventricular mass and volumes by magnetic resonance imaging," Journal of Computer Assisted Tomography, vol. 30, no. 3, pp. 426-432, 2006.

[41] F. Wiesmann, J. Ruff, K. H. Hiller, E. Rommel, A. Haase, and S. Neubauer, "Developmental changes of cardiac function and mass assessed with MRI in neonatal, juvenile, and adult mice," American Journal of Physiology - Heart and Circulatory Physiology, vol. 278, no. 2, pp. H652-H657, 2000.

[42] E. Heijman, J. P. Aben, C. Penners et al., "Evaluation of manual and automatic segmentation of the mouse heart from CINE MR images," Journal of Magnetic Resonance Imaging, vol. 27, no. 1, pp. 86-93, 2008.

[43] A. A. Young, H. Barnes, D. Davison, S. Neubauer, and J. E. Schneider, "Fast left ventricular mass and volume assessment in mice with three-dimensional guide-point modeling," Journal of Magnetic Resonance Imaging, vol. 30, no. 3, pp. 514-520, 2009.

[44] M. Hori, M. Inoue, and S. Fukui, "Correlation of ejection fraction and infarct size estimated from the total CK released in patients with acute myocardial infarction," British Heart Journal, vol. 41, no. 4, pp. 433-440, 1979.
[45] S. E. Litwin, S. E. Katz, J. P. Morgan, and P. S. Douglas, "Serial echocardiographic assessment of left ventricular geometry and function after large myocardial infarction in the rat," Circulation, vol. 89, no. 1, pp. 345-354, 1994.

[46] S. A. Rubin, M. C. Fishbein, and H. J. C. Swan, "Compensatory hypertrophy in the heart after myocardial infarction in the rat," Journal of the American College of Cardiology, vol. 1, no. 6, pp. 1435-1441, 1983.

[47] S. M. G. S. John and N. Sharpe, "Left ventricular remodeling after myocardial infarction: pathophysiology and therapy," Circulation, vol. 101, no. 25, pp. 2981-2988, 2000.

[48] J. P. M. Cleutjens, J. C. Kandala, E. Guarda, R. V. Guntaka, and K. T. Weber, "Regulation of collagen degradation in the rat myocardium after infarction," Journal of Molecular and Cellular Cardiology, vol. 27, no. 6, pp. 1281-1292, 1995.

[49] B. A. French and C. M. Kramer, "Mechanisms of postinfarct left ventricular remodeling," Drug Discovery Today, vol. 4, no. 3, pp. 185-196, 2007.

[50] A. C. S. Brau, L. W. Hedlund, and G. A. Johnson, "Cine magnetic resonance microscopy of the rat heart using cardiorespiratory-synchronous projection reconstruction," Journal of Magnetic Resonance Imaging, vol. 20, no. 1, pp. 31-38, 2004. 


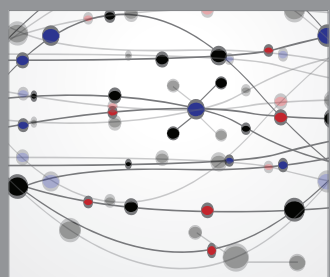

The Scientific World Journal
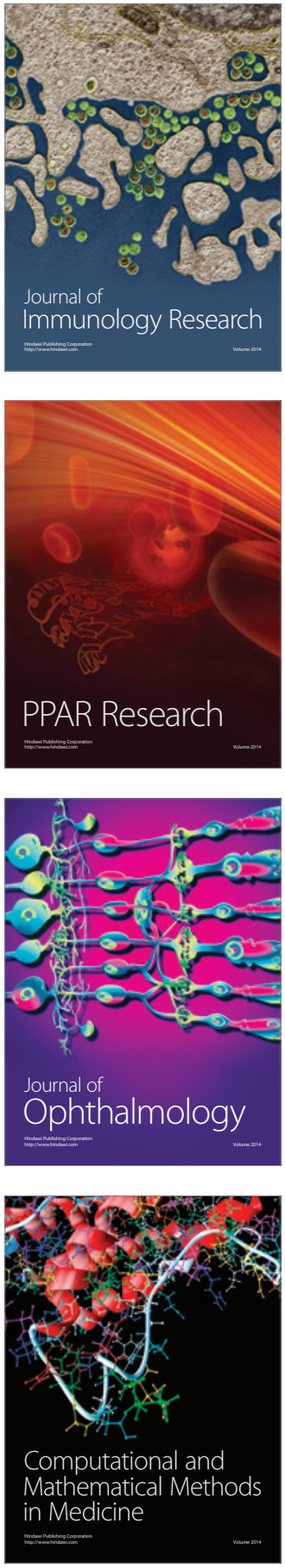

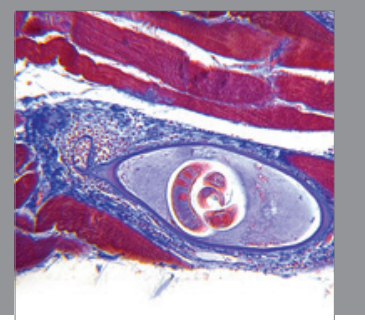

Gastroenterology

Research and Practice
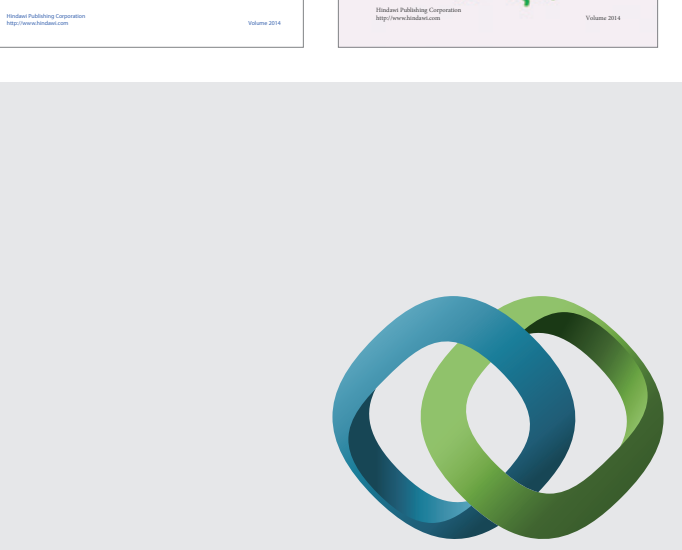

\section{Hindawi}

Submit your manuscripts at

http://www.hindawi.com
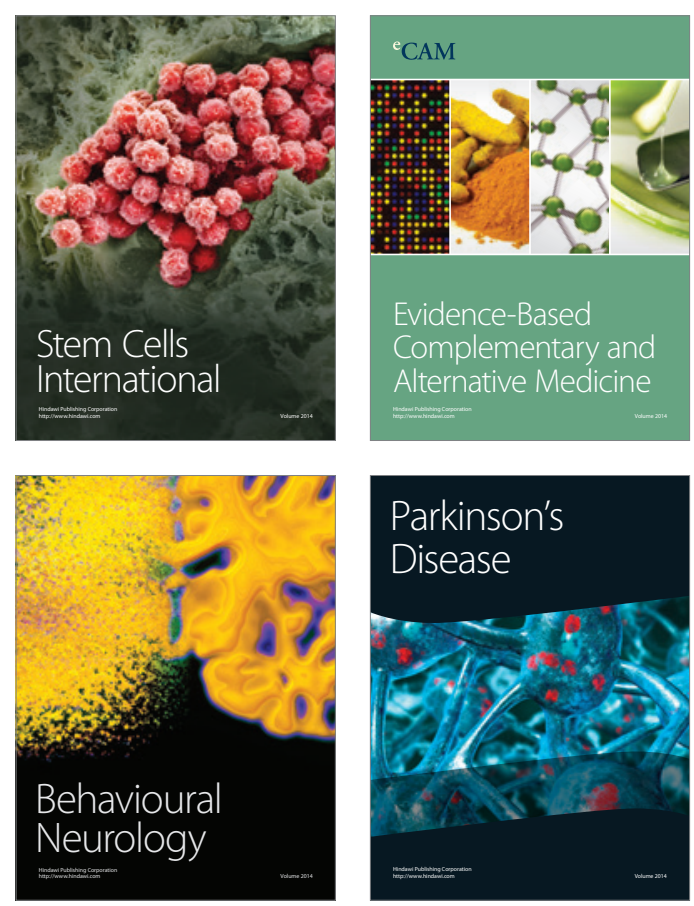

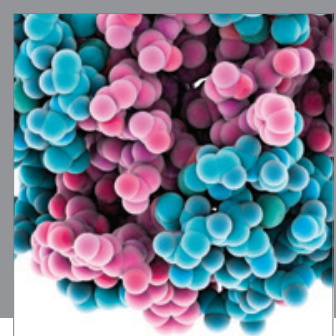

Journal of
Diabetes Research

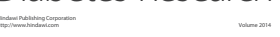

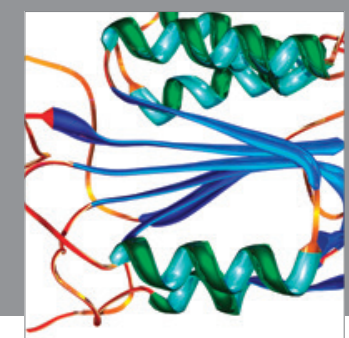

Disease Markers
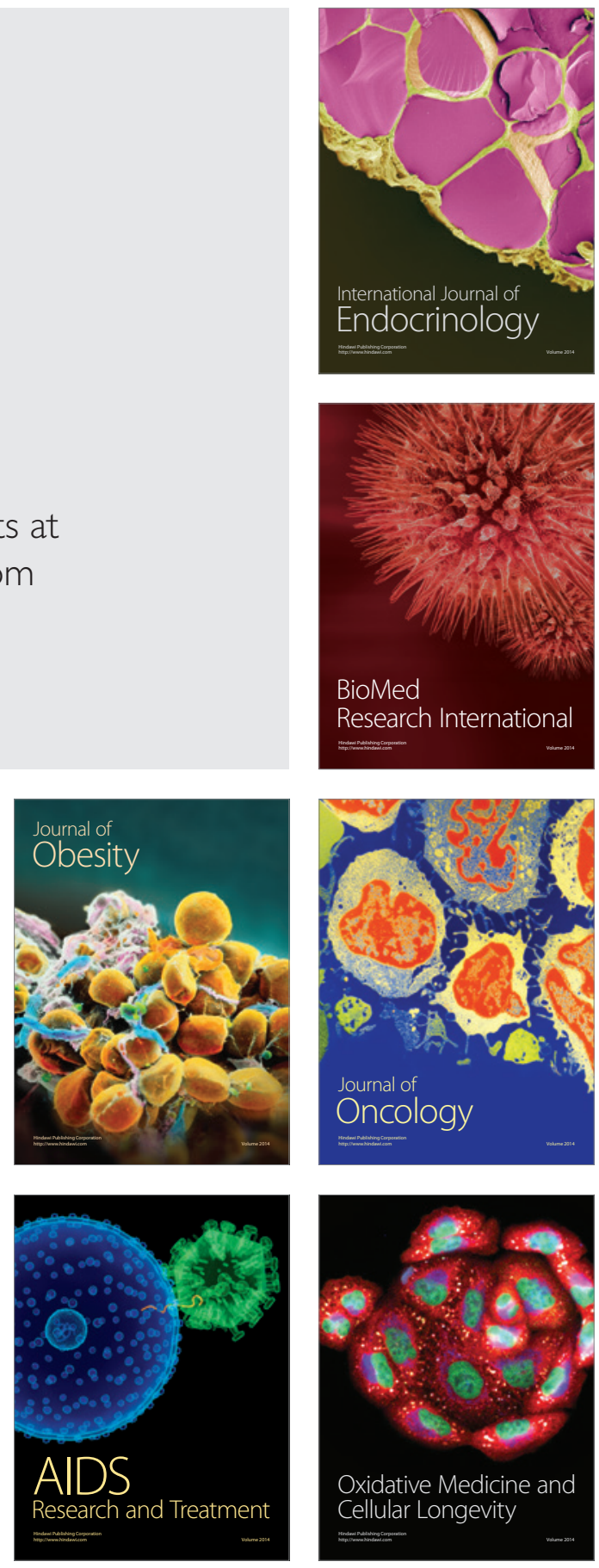